\title{
$k$-fold Sidon sets
}

\author{
Javier Cilleruelo* $\quad$ Craig Timmons ${ }^{\dagger}$ \\ Submitted: Nov 4, 2013; Accepted: Sep 30, 2014; Published: Oct 9, 2014 \\ Mathematics Subject Classifications: 05D99, 11B75
}

\begin{abstract}
Let $k \geqslant 1$ be an integer. A set $A \subset \mathbb{Z}$ is a $k$-fold Sidon set if $A$ has only trivial solutions to each equation of the form $c_{1} x_{1}+c_{2} x_{2}+c_{3} x_{3}+c_{4} x_{4}=0$ where $0 \leqslant\left|c_{i}\right| \leqslant k$, and $c_{1}+c_{2}+c_{3}+c_{4}=0$. We prove that for any integer $k \geqslant 1$, a $k$-fold Sidon set $A \subset[N]$ has at most $(N / k)^{1 / 2}+O\left((N k)^{1 / 4}\right)$ elements. Indeed we prove that given any $k$ positive integers $c_{1}<\cdots<c_{k}$, any set $A \subset[N]$ that contains only trivial solutions to $c_{i}\left(x_{1}-x_{2}\right)=c_{j}\left(x_{3}-x_{4}\right)$ for each $1 \leqslant i \leqslant j \leqslant k$, has at most $(N / k)^{1 / 2}+O\left(\left(c_{k}^{2} N / k\right)^{1 / 4}\right)$ elements. On the other hand, for any $k \geqslant 2$ we can exhibit $k$ positive integers $c_{1}, \ldots, c_{k}$ and a set $A \subset[N]$ with $|A| \geqslant\left(\frac{1}{k}+o(1)\right) N^{1 / 2}$, such that $A$ has only trivial solutions to $c_{i}\left(x_{1}-x_{2}\right)=c_{j}\left(x_{3}-x_{4}\right)$ for each $1 \leqslant i \leqslant j \leqslant k$.
\end{abstract}

Keywords: Sidon sets, $k$-fold Sidon sets

\section{Introduction}

Let $\Gamma$ be an abelian group. A set $A \subset \Gamma$ is a Sidon set if $a+b=c+d$ and $a, b, c, d \in$ $A$ implies $\{a, b\}=\{c, d\}$. Sidon sets in $\mathbb{Z}$ and in the group $\mathbb{Z}_{N}:=\mathbb{Z} / N \mathbb{Z}$ have been studied extensively. Erdős and Turán [5] proved that a Sidon set $A \subset[N]$ has at most $N^{1 / 2}+O\left(N^{1 / 4}\right)$ elements. Constructions of Singer [10], Bose and Chowla [2], and Ruzsa [9] show that this upper bound is asymptotically best possible. It is a prize problem of Erdős [4] to determine whether or not the error term is bounded. For more on Sidon sets we recommend O'Bryant's survey [8].

Let

$$
c_{1} x_{1}+\cdots+c_{r} x_{r}=0
$$

be an integer equation where $c_{i} \in \mathbb{Z} \backslash\{0\}$, and $c_{1}+\cdots+c_{r}=0$. Call such an equation an invariant equation. A solution $\left(x_{1}, \ldots, x_{r}\right) \in \mathbb{Z}^{r}$ to $(1)$ is trivial if there is a partition of $\{1, \ldots, r\}$ into nonempty sets $T_{1}, \ldots, T_{m}$ such that for every $1 \leqslant i \leqslant m$, we have

\footnotetext{
*Departamento de Matemáticas, Universidad Autónoma de Madrid, 28049 Madrid.

${ }^{\dagger}$ Department of Mathematics and Statistics, California State University Sacramento. Partially supported by NSF Grant DMS-1101489 through Jacques Verstraëte.
} 
$\sum_{j \in T_{i}} c_{j}=0$, and $x_{j_{1}}=x_{j_{2}}$ whenever $j_{1}, j_{2} \in T_{i}$. A natural extremal problem is to determine the maximum size of a set $A \subset[N]$ with only trivial solutions to (1). This problem was investigated in detail by Ruzsa [9]. One of the important open problems from [9] is the genus problem. Given an invariant equation $E: c_{1} x_{1}+\cdots+c_{r} x_{r}=0$, the genus $g(E)$ is the largest integer $m$ such that there is a partition of $\{1, \ldots, r\}$ into nonempty sets $T_{1}, \ldots, T_{m}$, such that $\sum_{j \in T_{i}} c_{j}=0$ for $1 \leqslant i \leqslant m$. Ruzsa proved that if $E$ is an invariant equation and $A \subset[N]$ has only trivial solutions to $E$, then $|A| \leqslant c_{E} N^{1 / g(E)}$. Here $c_{E}$ is a positive constant depending only on the equation $E$. Determining if there are sets $A \subset[N]$ with $|A|=N^{1 / g(E)-o(1)}$ and having only trivial solutions to $E$ is open for most equations. In particular, the genus problem is open for the equation $2 x_{1}+2 x_{2}=3 x_{3}+x_{4}$. This equation has genus 1 but the best known construction [9] gives a set $A \subset[N]$ with $|A| \geqslant c N^{1 / 2}$ where $c>0$ is a positive constant. More generally, Ruzsa showed that for any four variable equation $E: c_{1} x_{1}+c_{2} x_{2}=c_{3} x_{3}+c_{4} x_{4}$ with $c_{1}+c_{2}=c_{3}+c_{4}$ and $c_{i} \in \mathbb{N}$, there is a set $A \subset[N]$ with only trivial solutions to $E$ and $|A| \geqslant c_{E} N^{1 / 2-o(1)}$. In this paper we consider special types of four variable invariant equations.

Let $k \geqslant 1$ be an integer. A set $A \subset \mathbb{Z}$ is a $k$-fold Sidon set if $A$ has only trivial solutions to each equation of the form

$$
c_{1} x_{1}+c_{2} x_{2}+c_{3} x_{3}+c_{4} x_{4}=0
$$

where $0 \leqslant\left|c_{i}\right| \leqslant k$, and $c_{1}+c_{2}+c_{3}+c_{4}=0$. A 1 -fold Sidon set is a Sidon set. A 2-fold Sidon set has only trivial solutions to each of the equations

$$
x_{1}+x_{2}-x_{3}-x_{4}=0, \quad 2 x_{1}+x_{2}-2 x_{3}-x_{4}=0, \quad 2 x_{1}-x_{2}-x_{3}=0 .
$$

One can also define $k$-fold Sidon sets in $\mathbb{Z}_{N}$. We must add the condition that $N$ is relatively prime to all integers in the set $\{1,2, \ldots, k\}$. The reason for this is that if a coefficient $c_{i} \in\{1,2, \ldots, k\}$ has a common factor with $N$, then in $\mathbb{Z}_{N}$ one could have $c_{i}\left(a_{1}-a_{2}\right)=0$ with $a_{1} \neq a_{2}$. In this case, if $|A| \geqslant 3$, we can choose $a_{3} \in A \backslash\left\{a_{1}, a_{2}\right\}$, and obtain the nontrivial solution $\left(x_{1}, x_{2}, x_{3}, x_{4}\right)=\left(a_{1}, a_{2}, a_{3}, a_{3}\right)$ to the equation $c_{i}\left(x_{1}-x_{2}\right)+x_{3}-x_{4}=0$.

Lazebnik and Verstraëte [6] were the first to define $k$-fold Sidon sets. They conjectured the following.

Conjecture 1 (Lazebnik, Verstraëte [6]). For any integer $k \geqslant 3$, there is a positive constant $c_{k}>0$ such that for all integers $N \geqslant 1$, there is a $k$-fold Sidon set $A \subset[N]$ with $|A| \geqslant c_{k} N^{1 / 2}$.

This conjecture is still open. Lazebnik and Verstraëte proved that for infinitely many $N$, there is a 2 -fold Sidon set $A \subset \mathbb{Z}_{N}$ with $|A| \geqslant \frac{1}{2} N^{1 / 2}-3$. Axenovich [1] and Verstraëte (unpublished) observed that one can adapt Ruzsa's construction for four variable equations (Theorem 7.3, [9]) to construct $k$-fold Sidon sets $A \subset[N]$ or $A \subset \mathbb{Z}_{N}$ with $|A| \geqslant c_{k} N^{1 / 2} e^{-c_{k} \sqrt{\log N}}$ for any $k \geqslant 3$. An affirmative answer to Conjecture 1 , even in the case when $k=3$, would have applications to hypergraph Turán problems [6] and extremal graph theory [11]. 
Since any $k$-fold Sidon set is a Sidon set, the trivial upper bound $|A| \leqslant \sqrt{N-3 / 4}+1 / 2$ for a Sidon set $A \subset \mathbb{Z}_{N}$, and the Erdős-Turán bound $|A| \leqslant N^{1 / 2}+O\left(N^{1 / 4}\right)$ for any Sidon set $A \subset[N]$, also hold for $k$-fold Sidon sets. We will obtain better upper bounds for $k$-fold Sidon sets. Instead of considering all the possible equations $c_{1} x_{1}+c_{2} x_{2}+c_{3} x_{3}+c_{4} x_{4}=0$ with $c_{1}+c_{2}+c_{3}+c_{4}=0$, we will take advantage only of the equations of the form

$$
c_{1}\left(x_{1}-x_{2}\right)=c_{2}\left(x_{3}-x_{4}\right) .
$$

For any $c_{1}, \ldots, c_{k}$ with $\left(c_{i}, N\right)=1$, if $A \subset \mathbb{Z}_{N}$ contains only trivial solutions to $c_{i}\left(x_{1}-x_{2}\right)=c_{j}\left(x_{3}-x_{4}\right)$ for each $1 \leqslant i \leqslant j \leqslant k$, then

$$
|A| \leqslant \sqrt{\frac{N-1}{k}+\frac{1}{4}}+\frac{1}{2}
$$

To see this, consider all elements of the form $c_{i}(x-y)$ where $1 \leqslant i \leqslant k$, and $x \neq y$ are elements of $A$. All of these elements are distinct and nonzero. Therefore, $k|A|(|A|-1) \leqslant$ $N-1$ which is equivalent to (2).

The short counting argument used to obtain (2) does not work in $\mathbb{Z}$. Using a more sophisticated argument, we can show that a bound similar to (2) does hold in $\mathbb{Z}$.

Theorem 2. Let $k \geqslant 1$ be an integer and $1 \leqslant c_{1}<c_{2}<\cdots<c_{k}$ be a set of $k$ distinct integers. If $A \subset[N]$ is a set with only trivial solutions to $c_{i}\left(x_{1}-x_{2}\right)=c_{j}\left(x_{3}-x_{4}\right)$ for each $1 \leqslant i \leqslant j \leqslant k$, then

$$
|A| \leqslant\left(\frac{N}{k}\right)^{1 / 2}+O\left(\left(\frac{c_{k}^{2} N}{k}\right)^{1 / 4}\right) .
$$

Taking $c_{j}=j$ for $1 \leqslant j \leqslant k$, we have the following corollary.

Corollary 3. If $k \geqslant 1$ is an integer and $A \subset[N]$ is a $k$-fold Sidon set, then

$$
|A| \leqslant\left(\frac{N}{k}\right)^{1 / 2}+O\left((k N)^{1 / 4}\right) .
$$

In Theorem 2 and Corollary 3, the Landau symbols are with respect to $N$. That is, we view $k, c_{1}, \ldots, c_{k}$ as being fixed, and $N$ tending to infinity.

It is natural to ask if we can improve Corollary 3 if we make full use of the assumption that $A$ is a $k$-fold Sidon set. For example, the bound $|A| \leqslant(N / 3)^{1 / 2}+O\left(N^{1 / 4}\right)$ holds under the assumption that $A \subset[N]$ has only trivial solutions to $c_{1}\left(x_{1}-x_{2}\right)=c_{2}\left(x_{3}-x_{4}\right)$ for each $1 \leqslant c_{1} \leqslant c_{2} \leqslant 3$. A 3 -fold Sidon set additionally has only trivial solutions to $2 x_{1}+2 x_{2}=3 x_{3}+x_{4}$. Our argument does not capture this property. It is not known if this additional assumption would improve the upper bound $|A| \leqslant(N / 3)^{1 / 2}+O\left(N^{1 / 4}\right)$.

The method used by Lazebnik and Verstraëte to construct 2-fold Sidon sets is rather robust. Using this method, we prove the following theorem. 
Theorem 4. There exist $k$ distinct integers $c_{1}, \ldots, c_{k}$ and infinitely many $N$, such that there is a set $A \subset \mathbb{Z}_{N}$ with

$$
|A| \geqslant \frac{N^{1 / 2}}{k}(1-o(1))
$$

and having only trivial solutions to $c_{i}\left(x_{1}-x_{2}\right)=c_{j}\left(x_{3}-x_{4}\right)$ for each $1 \leqslant i \leqslant j \leqslant k$.

The next section contains the proof of Theorem 2. Section 3 contains the proof of Theorem 4.

\section{Proof of Theorem 2}

For finite sets $B, C \subset \mathbb{Z}$, define

$$
r_{B+C}(x)=|\{(b, c): b+c=x, b \in B, c \in C\}|
$$

and

$$
r_{B-C}(x)=|\{(b, c): b-c=x, b \in B, c \in C\}| .
$$

The following useful lemma has appeared in the literature (see [3] or [9]).

Lemma 5. For any finite sets $B, C \subset \mathbb{Z}$,

$$
\frac{(|B||C|)^{2}}{|B+C|} \leqslant|B||C|+\sum_{x \neq 0} r_{B-B}(x) r_{C-C}(x) .
$$

Proof. Observe $\sum_{x \in B+C} r_{B+C}(x)$ counts every ordered pair $(b, c) \in B \times C$ exactly once so that $|B||C|=\sum_{x \in B+C} r_{B+C}(x)$. By the Cauchy-Schwarz inequality,

$$
\frac{(|B||C|)^{2}}{|B+C|}=\frac{\left(\sum_{x \in B+C} r_{B+C}(x)\right)^{2}}{|B+C|} \leqslant \sum_{x} r_{B+C}^{2}(x) .
$$

The sum $\sum_{x} r_{B+C}^{2}(x)$ counts 4 -tuples $\left(b, b^{\prime}, c, c^{\prime}\right)$ with $b, b^{\prime} \in B, c, c^{\prime} \in C$, and $b+c=b^{\prime}+c^{\prime}$. The equation $b+c=b^{\prime}+c^{\prime}$ is equivalent to $b-b^{\prime}=c^{\prime}-c$ and so

$$
\sum_{x} r_{B+C}^{2}(x)=\sum_{x} r_{B-B}(x) r_{C-C}(x)
$$

Combining (4) and (5), we have

$$
\frac{(|B||C|)^{2}}{|B+C|} \leqslant \sum_{x} r_{B-B}(x) r_{C-C}(x)=|B||C|+\sum_{x \neq 0} r_{B-B}(x) r_{C-C}(x)
$$

which proves (3). 
Before giving the proof of Theorem 2 we take a moment to describe some of ideas of the proof. Suppose $A \subset[N]$ is a set satisfying the hypothesis of Theorem 2. If $c_{r} A:=\left\{c_{r} a: a \in A\right\}$, then the $k$-fold Sidon property implies that

$$
\sum_{r=1}^{k} r_{c_{r} A-c_{r} A}(y) \leqslant 1
$$

for any $y \neq 0$. One may then apply Lemma 5 to $c_{r} A$ and the interval $C=\{0,1, \ldots, m-1\}$ for each $1 \leqslant r \leqslant k$. An obstacle in this approach is the expression $\left|c_{r} A+C\right|$ that appears in the denominator on the left hand side of (3). Since $c_{r} A+C \subset\left\{1,2, \ldots, c_{r} N+m-1\right\}$, we have $\left|c_{r} A+C\right| \leqslant c_{r} N+m$ but this upper bound that is too large for our approach. Instead of $c_{r} A$, we will consider the sets $B_{r, i}:=\left\{x: c_{r} x+i \in A\right\}, 0 \leqslant i \leqslant c_{r}-1$. We will show that an analogue of (6) holds for the $B_{r, i}$ 's which, although not difficult, is one of the most important parts of the proof. Additionally, we have that $\left|B_{r, i}+C\right| \leqslant N / c_{r}+m$ and this is what leads to a more effective application of Lemma 5.

Proof of Theorem 2. Let $1 \leqslant c_{1}<c_{2}<\cdots<c_{k}$ be $k$ distinct integers. Let $A \subset[N]$ be a set with only trivial solutions to $c_{i}\left(x_{1}-x_{2}\right)=c_{j}\left(x_{3}-x_{4}\right)$ for each $1 \leqslant i \leqslant j \leqslant k$. Let

$$
B_{r, i}=\left\{x: c_{r} x+i \in A\right\}
$$

for $1 \leqslant r \leqslant k$ and $0 \leqslant i \leqslant c_{r}-1$. Therefore,

$$
|A|=\sum_{i=0}^{c_{r}-1}\left|\left\{a \in A: a \equiv i \quad\left(\bmod c_{r}\right)\right\}\right|=\sum_{i=0}^{c_{r}-1}\left|B_{r, i}\right|
$$

so by the Cauchy-Schwarz inequality,

$$
|A|^{2}=\left(\sum_{i=0}^{c_{r}-1}\left|B_{r, i}\right|\right)^{2} \leqslant c_{r} \sum_{i=0}^{c_{r}-1}\left|B_{r, i}\right|^{2}
$$

For any $y \neq 0$,

$$
\sum_{r=1}^{k} \sum_{i=0}^{c_{r}-1} r_{B_{r, i}-B_{r, i}}(y) \leqslant 1
$$

To see this, suppose

$$
y=x_{1}-x_{2}=x_{3}-x_{4}
$$

where $x_{1}, x_{2} \in B_{r, i}$ and $x_{3}, x_{4} \in B_{r^{\prime}, i^{\prime}}$ for some $1 \leqslant r, r^{\prime} \leqslant k, 1 \leqslant i \leqslant c_{r}-1$, and $1 \leqslant i^{\prime} \leqslant c_{r^{\prime}}-1$. There are elements $a_{1}, a_{2}, a_{3}, a_{4} \in A$ such that

$$
c_{r} x_{1}+i=a_{1}, \quad c_{r} x_{2}+i=a_{2}, \quad c_{r^{\prime}} x_{3}+i^{\prime}=a_{3}, \quad \text { and } \quad c_{r^{\prime}} x_{4}+i^{\prime}=a_{4} .
$$

Then (9) implies

$$
\frac{1}{c_{r}}\left(a_{1}-i\right)-\frac{1}{c_{r}}\left(a_{2}-i\right)=\frac{1}{c_{r^{\prime}}}\left(a_{3}-i^{\prime}\right)-\frac{1}{c_{r^{\prime}}}\left(a_{4}-i^{\prime}\right),
$$


thus $c_{r^{\prime}}\left(a_{1}-a_{2}\right)=c_{r}\left(a_{3}-a_{4}\right)$. Since $y \neq 0$, we have $a_{1} \neq a_{2}$ and $a_{3} \neq a_{4}$ and then we would have a non trivial solution to the equation $c_{r^{\prime}}\left(x_{1}-x_{2}\right)=c_{r}\left(x_{3}-x_{4}\right)$.

Let $C=\{0,1, \ldots, m-1\}$. For any $1 \leqslant r \leqslant k$ and $0 \leqslant i \leqslant c_{r}-1$, the set $B_{r, i}+C$ is contained in the interval $\left\{0,1, \ldots,\left\lfloor N / c_{r}\right\rfloor+m-1\right\}$. This gives the trivial estimate $\left|B_{r, i}+C\right| \leqslant N / c_{r}+m$. By Lemma 5 applied to $B_{r, i}$ and $C$,

$$
\frac{\left|B_{r, i}\right|^{2} m^{2}}{N / c_{r}+m} \leqslant\left|B_{r, i}\right| m+\sum_{y \neq 0} r_{B_{r, i}-B_{r, i}}(y) r_{C-C}(y) .
$$

We sum this inequality over all $1 \leqslant r \leqslant k$ and $0 \leqslant i \leqslant c_{r}-1$ to get

$$
\begin{aligned}
m^{2} \sum_{r=1}^{k} \frac{1}{N / c_{r}+m} \sum_{i=0}^{c_{r}-1}\left|B_{r, i}\right|^{2} & \leqslant \sum_{r=1}^{k} \sum_{i=0}^{c_{r}-1}\left|B_{r, i}\right| m \\
& +\sum_{y \neq 0} \sum_{r=1}^{k} \sum_{i=0}^{c_{r}-1} r_{B_{r, i}-B_{r, i}}(y) r_{C-C}(y) \\
& \leqslant k|A| m+\sum_{y \neq 0} r_{C-C}(y) \\
& \leqslant m(k|A|+m) .
\end{aligned}
$$

From (7) and the previous inequality, we deduce that

$$
m^{2}|A|^{2} \sum_{r=1}^{k} \frac{1}{N+c_{r} m} \leqslant m(k|A|+m) .
$$

The left hand side of (10) is at least $\frac{|A|^{2} k m^{2}}{N+c_{k} m}$. Therefore, $\frac{|A|^{2} k m}{N+c_{k} m} \leqslant k|A|+m$, and so

$$
|A|^{2} k m \leqslant\left(N+c_{k} m\right)(m+k|A|) .
$$

We complete the square and use the inequality $(x+y)^{2} \leqslant 2 x^{2}+2 y^{2}$ to obtain

$$
\begin{aligned}
\left(|A|-\left(\frac{N}{2 m}+\frac{c_{k}}{2}\right)\right)^{2} & \leqslant \frac{N}{k}+\frac{c_{k} m}{k}+\left(\frac{N}{2 m}+\frac{c_{k}}{2}\right)^{2} \\
& \leqslant \frac{N}{k}+\frac{c_{k} m}{k}+\frac{N^{2}}{2 m^{2}}+\frac{c_{k}^{2}}{2} \\
& =\frac{N}{k}\left(1+\frac{c_{k} m}{N}+\frac{N k}{2 m^{2}}+\frac{k c_{k}^{2}}{2 N}\right) .
\end{aligned}
$$

Taking square roots and using the inequality $\sqrt{1+x} \leqslant 1+x$ for $x \geqslant 0$, we solve for $|A|$ to get

$$
\begin{aligned}
|A| & \leqslant\left(\frac{N}{k}\right)^{1 / 2}\left(1+\frac{c_{k} m}{N}+\frac{N k}{2 m^{2}}+\frac{k c_{k}^{2}}{2 N}\right)+\frac{N}{2 m}+\frac{c_{k}}{2} \\
& =\left(\frac{N}{k}\right)^{1 / 2}+\frac{c_{k} m}{k^{1 / 2} N^{1 / 2}}+\frac{N^{3 / 2} k^{1 / 2}}{2 m^{2}}+\frac{k^{1 / 2} c_{k}^{2}}{2 N^{1 / 2}}+\frac{N}{2 m}+\frac{c_{k}}{2} .
\end{aligned}
$$


Take $m=\left\lceil\left(N^{3 / 4} k^{1 / 4}\right) / c_{k}^{1 / 2}\right\rceil$ to get $|A| \leqslant\left(\frac{N}{k}\right)^{1 / 2}+O\left(\left(c_{k}^{2} N / k\right)^{1 / 4}\right)$. This completes the proof of Theorem 2 .

\section{Proof of Theorem 4}

Let $k \geqslant 2$ be an integer. Let $p$ be a prime, and let $M \geqslant 1$ be a large integer. Let $r$ be any prime with $r>M k$. Let $i \geqslant 1$ be an integer, and set $t=r^{i}$ and $q=p^{t}$.

We will prove that for $c_{j}=p^{j-1}$ for $j=1, \ldots k$ there exists a set $A \subset \mathbb{Z}_{q^{2}-1}$ with $|A| \geqslant \frac{q}{k}\left(1-\frac{1}{M}\right)-\left(p^{4}-1\right)(M-1)$ and having only trivial solutions to

$$
x_{1}-x_{2}=p^{j-1}\left(x_{3}-x_{4}\right)
$$

for $1 \leqslant j \leqslant k$. This proves Theorem 4 because as $i$ tends to infinity, the term $\frac{q}{k}\left(1-\frac{1}{M}\right)$ is the dominant term. $M$ can be taken as large as we want, and $\left(p^{4}-1\right)(M-1)$ is constant with respect to $i$.

Let $\theta$ be a generator of the cyclic group $\mathbb{F}_{q^{2}}^{*}$. Bose and Chowla [2] proved that the set

$$
C(q, \theta)=\left\{a \in \mathbb{Z}_{q^{2}-1}: \theta^{a}-\theta \in \mathbb{F}_{q}\right\}
$$

is a Sidon set in $\mathbb{Z}_{q^{2}-1}$. Lindström [7] proved

$$
B(q, \theta)=\left\{b \in \mathbb{Z}_{q^{2}-1}: \theta^{b}+\theta^{q b}=1\right\}
$$

is a translate of $C(q, \theta)$ and is therefore a Sidon set.

Lemma 6. The map $x \mapsto p x$ is an injection from $\mathbb{Z}_{q^{2}-1}$ to $\mathbb{Z}_{q^{2}-1}$ that maps $B(q, \theta)$ to $B(q, \theta)$.

Proof. The map $x \mapsto p x$ is 1 -to- 1 since $p$ is relatively prime to $q^{2}-1$. If $b \in B(q, \theta)$, then

$$
1=\left(\theta^{b}+\theta^{q b}\right)^{p}=\theta^{p b}+\theta^{q(p b)}
$$

so $p b \in B(q, \theta)$.

Let $\pi: B(q, \theta) \rightarrow B(q, \theta)$ be the permutation $\pi(b)=p b$. As in [6], we use the cycles of $\pi$ to define $A$. Let $\sigma=\left(b_{1}, \ldots, b_{m}\right)$ be a cycle of $\pi$. If $m<k$, then remove all elements of $\sigma$ from $B(q, \theta)$. If $m \geqslant k$, then remove all $b_{j}$ in $\sigma$ for which $j$ is not divisible by $k$. Do this for each cycle of $\pi$. Let $A$ be the resulting subset of $B(q, \theta)$.

Lemma 7. For each $c \in\left\{1, p, p^{2}, \ldots, p^{k-1}\right\}, A$ has only trivial solutions to

$$
x_{1}-x_{2}=c\left(x_{3}-x_{4}\right) \text {. }
$$


Proof. Suppose $a_{1}, a_{2}, a_{3}, a_{4} \in A$ and $a_{1}-a_{2}=p^{j}\left(a_{3}-a_{4}\right)$ for some $0 \leqslant j \leqslant k-1$. By Lemma 6 , there are elements $b_{3}, b_{4} \in B(q, \theta)$ such that $p^{j} a_{3}=b_{3}$ and $p^{j} a_{4}=b_{4}$. This gives $a_{1}-a_{2}=b_{3}-b_{4}$. Since $B(q, \theta)$ is a Sidon set, either $a_{1}=a_{2}, b_{3}=b_{4}$ or $a_{1}=b_{3}$, $a_{2}=b_{4}$.

If $a_{1}=a_{2}$ and $b_{3}=b_{4}$, then $a_{3}=a_{4}$ and the solution $\left(a_{1}, a_{2}, a_{3}, a_{4}\right)$ is trivial. Suppose $a_{1}=b_{3}$ and $a_{2}=b_{4}$. This implies $b_{3} \in A$, so both $p^{j} a_{3}$ and $a_{3}$ are in $A$. This contradicts the way in which $A$ was constructed.

Lemma 8. $|A| \geqslant \frac{q}{k}\left(1-\frac{1}{M}\right)-\left(p^{4}-1\right)(M-1)$.

Proof. In order to obtain a lower bound on $|A|$, we need to estimate the number of cycles of $\pi$ that are short. For instance, if all cycles of $\pi$ have length less than $k$, then $|A|=0$. For a cycle $\sigma$ of $\pi$ with length $m k \geqslant M k$, we delete at most $m(k-1)$ elements from $B(q, \theta)$ and keep at least $m-1$ elements.

We estimate the number of cycles of length at most $M k-1$. Let $\sigma=\left(b, p b, \ldots, p^{e-1} b\right)$ be a cycle of $\pi$ of length $e$ where $e \leqslant M k-1$. The integer $e$ is the smallest positive integer such that $p^{e} b \equiv b\left(\bmod q^{2}-1\right)$. This is the same as saying that the order of $p$ in the multiplicative group of units $\mathbb{Z}_{n}^{*}$ is $e$ where $n=\frac{q^{2}-1}{\operatorname{gcd}\left(b, q^{2}-1\right)}$. Since

$$
p^{4 t}-1=\left(p^{2 t}-1\right)\left(p^{2 t}+1\right)=\left(q^{2}-1\right)\left(p^{2 t}+1\right)
$$

we have $p^{4 t} \equiv 1\left(\bmod q^{2}-1\right)$, so $e$ must divide $4 t=4 r^{i}$. Since $r$ is prime and $r \geqslant M k, e$ cannot divide $r$, so $e$ must divide 4 . To count the number of cycles of $\pi$ with length at most $M k-1$, it is enough to count the elements $x \in \mathbb{Z}_{q^{2}-1} \backslash\{0\}$ such that $p^{4} x \equiv x\left(\bmod q^{2}-1\right)$. This follows from the fact that if $e \in\{1,2\}$ and $p^{e} x \equiv x\left(\bmod q^{2}-1\right)$, then $p^{4} x \equiv$ $x\left(\bmod q^{2}-1\right)$. The number of solutions to this congruence is $\operatorname{gcd}\left(p^{4}-1, q^{2}-1\right) \leqslant p^{4}-1$. Therefore, there are at most $p^{4}-1$ cycles of $\pi$ of length at most $M k-1$. For a cycle of length at least $M k$, the proportion of elements of the cycle that are put into $A$ is at least $\frac{M-1}{M k}$ (the function $f(x)=\frac{x-1}{x k}$ is increasing provided $k>0$ ). Since $|B(q, \theta)|=q$,

$$
|A| \geqslant\left(q-\left(p^{4}-1\right) M k\right)\left(\frac{M-1}{M k}\right)=\frac{q}{k}\left(1-\frac{1}{M}\right)-\left(p^{4}-1\right)(M-1) .
$$

Theorem 4 follows from Lemmas 7 and 8 .

\section{Concluding Remarks}

The most important open problem concerning $k$-fold Sidon sets is an answer to Conjecture 1. The case $k=3$ is particularly interesting. A 3-fold Sidon set $A \subset[N]$ with $|A| \geqslant c N^{1 / 2}$ is known to imply the existence of a graph with $c_{1} N$ vertices, $c_{2} N^{3 / 2}$ edges, and every edge is in exactly one cycle of length four [11].

Another problem is to determine the maximum size of a 2-fold Sidon set in $\mathbb{Z}_{N}$ or $[N]$. Let $S_{k}(N)$ be the maximum size of a $k$-fold Sidon set in $\mathbb{Z}_{N}$. For any integer $t \geqslant 1$, 
there are 2-fold Sidon sets $A \subset \mathbb{Z}_{N}, N=2^{2^{t+1}}+2^{2^{t}}+1$, with $|A| \geqslant \frac{1}{2} N^{1 / 2}-3$ (see [6]). Theorem 2 gives an upper bound of $(N / 2)^{1 / 2}+O\left(N^{1 / 4}\right)$ so

$$
\frac{1}{2} \leqslant \limsup _{N \rightarrow \infty} \frac{S_{2}(N)}{N^{1 / 2}} \leqslant \frac{1}{2^{1 / 2}}
$$

It would be interesting to determine the above limit. In the case of Sidon sets, we have $\lim \sup _{N \rightarrow \infty} \frac{S_{1}(N)}{N^{1 / 2}}=1$ by [5] and [10].

\section{References}

[1] M. Axenovich, personal communication.

[2] R. C. Bose, S. Chowla, Theorems in the additive theory of numbers, Comment. Math. Helv. 37 (1962/1963), 141-147.

[3] J. Cilleruelo, Sidon sets in $\mathbb{N}^{d}$, J. Combin. Theory, Series A 117 (2010) 857-871.

[4] P. Erdös, A survey of problems in combinatorial number theory, Annals of Discrete Mathematics 6 (1980), 89-115.

[5] P. Erdős, P. Turán, On a problem of Sidon in additive number theory, and on some related results, Journal of the London Mathematical Society, 16 (1941).

[6] F. Lazebnik, J. Verstraëte, On hypergraphs of girth five, Electronic J. of Combinatorics, 10, (2003), \#R25.

[7] B. Lindström, A translate of Bose-Chowla $B_{2}$-sets, Studia Sc. Math. Hungar., 36, (2000), 331-333.

[8] K. O'Bryant, A complete annotated bibliography of work related to Sidon sequences, Electronic J. of Combinatorics DS 11 (2004).

[9] I. Ruzsa, Solving a linear equation in a set of integers I, Acta Arith. 653 (1993), 259-282.

[10] J. Singer, A theorem in finite projective geometry and some applications to number theory, Trans. Amer. Math. Soc. 43 (1938), 377-385.

[11] C. Timmons, J. Verstraëte, A counterexample to sparse removal, submitted. arXiv:1312.2994. 